\title{
Keratosis obturans y colesteatoma del conducto auditivo externo: Caso clínico y revisión de la literatura
}

\author{
Keratosis obturans and external auditory canal cholesteatoma: \\ Case report and review of the literature
}

\author{
Andrés Alvo V', Natalia Jara $\mathbf{0}^{2}$, Fabián Rubio $\mathbf{M}^{1}$, Carlos Stott $\mathbf{C}^{1}$, Alfredo Naser G1.
}

\begin{abstract}
RESUMEN
La keratosis obturans y el colesteatoma del conducto auditivo externo son dos condiciones caracterizadas por la acumulación patológica de material queratinizado en el oído externo. El primero se presenta con un crecimiento circunferencial que ocluye y remodela el conducto, usualmente sin erosionarlo, mientras que el segundo se caracteriza por erosionar un área del conducto, con necrosis y secuestro óseo. El diagnóstico diferencial entre estas dos entidades es importante, ya que difieren en la presentación clínica, el comportamiento biológico y las complicaciones asociadas. Esto puede verse reflejado en cambios en el manejo terapéutico de estos pacientes.
\end{abstract}

Palabras clave: Keratosis obturans, colesteatoma, conducto auditivo externo.

\begin{abstract}
Keratosis obturans and external auditory canal cholesteatoma are two conditions characterized by the pathological accumulation of keratinized debris in the external ear. The former shows a circumferential growth that occludes and remodels the canal, usually without erosion, whereas the latter is characterized by erosion of part of the canal, with necrosis and bone sequestration. Establishing a differential diagnosis between both diseases is important, because they differ in clinical presentation, biological behavior and associated complications. This can be reflected in the therapeutic management of these patients.
\end{abstract}

Key words: Keratosis obturans, cholesteatoma, external auditory canal.

\footnotetext{
${ }^{1}$ Servicio de Otorrinolaringología, Hospital Clínico Universidad de Chile.

${ }^{2}$ Médico Cirujano. Magíster en Ciencias Médicas, Universidad de Chile.
} 


\section{INTRODUCCIÓN}

La keratosis obturans (KO) y el colesteatoma del CAE (CC) son dos entidades patológicas caracterizadas por la acumulación de descamaciones epiteliales queratinizadas en el conducto auditivo externo (CAE).

La descripción inicial realizada por Toynbee en 1850 no distinguía a la KO del CC como dos patologías distintas 1 . En 1980, Piepergerdes y cols publicaron un artículo comparando hallazgos clínicos y patológicos, realizando la distinción entre ambas. Mientras que la KO se presenta como un tapón de material queratinizado en el CAE, el CC se caracteriza por una invasión local erosiva de tejido epitelial hacia el hueso?

Dichas diferencias tienen importancia en el diagnóstico, evolución y manejo de estos pacientes; por lo que deben ser conocidas por el otorrinolaringólogo. Presentamos a continuación un caso clínico que ilustra los aspectos relevantes respecto a la clínica y tratamiento, y una revisión exhaustiva de la literatura.

\section{CASO CLÍNICO}

Paciente de 39 años sin antecedentes mórbidos, con historia de otorrea indolora de larga data del

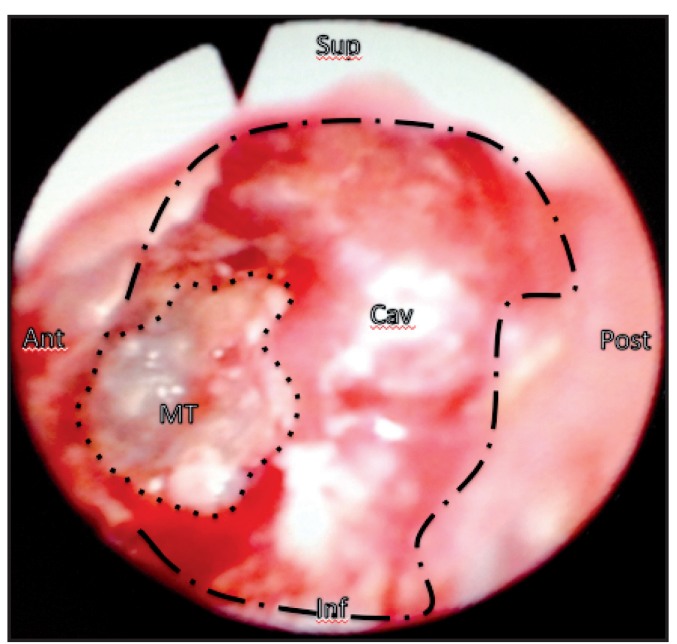

Figura 1. Otoscopia del oído izquierdo, inmediatamente después del desbridamiento. Ant: anterior, Sup: superior, Post: posterior, Inf: inferior, MT: membrana timpánica, Cav: cavidad resultante de la erosión en el CAE por el CC, Línea punteada: límite de la MT, Línea interrumpida: límite de la cavidad erosionada. oído izquierdo sin control previo. Consultó por una paresia facial periférica izquierda House-Brackmann 4 de un día de evolución, con otorrea y otalgia del oído izquierdo.

Al examen físico se observó la ocupación completa del CAE por material de aspecto epitelial, con otorrea blanquecina de mal olor. Se realizó aseo cuidadoso bajo otomicroscopía, identificándose una erosión del CAE óseo en sus paredes posterosuperior e inferior; la membrana timpánica se encontraba indemne (Figura 1). Se logró un adecuado desbridamiento en esta instancia, logrando un buen control de la cavidad, manteniendo la zona correspondiente al nervio facial a la vista.

Se solicitó una tomografía computarizada (TC) de oídos, que evidenció una masa con densidad de partes blandas en el CAE asociada a erosión ósea en el conducto óseo que comprometía la porción mastoidea del nervio facial (Figura 2). Con estos antecedentes se estableció el diagnóstico de colesteatoma del CAE, estadio III según la clasificación de Naim y cols (Tabla 1). Esto debido a que a pesar de haber paresia facial, clínica y radiológicamente se comportó como inflamatoria, debido a la dehis-

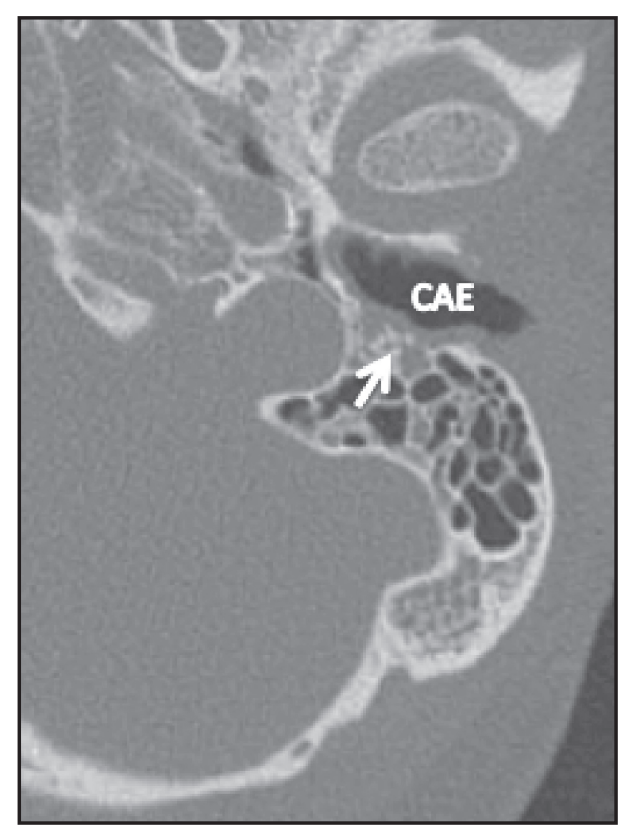

Figura 2. TC de oídos, corte axial. La flecha muestra la dehiscencia ósea del CAE que compromete la porción mastoidea del nervio facial izquierdo. El colesteatoma ya había sido retirado del conducto. 
Tabla 1. Clasificación del colesteatoma del CAE (adaptado de Naim y cols ${ }^{13}$ )

\begin{tabular}{|ll|}
\hline Estadio & Definición \\
\hline I I & Hiperplasia e hiperemia del epitelio del CAE. Aumento de la tasa de apoptosis en la región superior. \\
& Inflamación localizada del epitelio hiperproliferado con periostitis adyacente. Sin destrucción del CAE óseo. \\
& Acumulación de detritus de queratina. Clínicamente presenta dolor sordo y, en caso de sobreinfección, \\
& otorrea. \\
a. Superficie epitelial intacta sin compromiso del canal óseo & \\
& b. Excavación del epitelio defectuoso con compromiso del canal \\
III & Destrucción del CAE óseo con hueso secuestrado (osteonecrosis aséptica). Excavación del epitelio hacia el \\
& hueso adyacente. Acumulación de detritus de queratina con posible sobreinfección y otorrea. \\
IV & Destrucción espontánea de estructuras anatómicas adyacentes (subclases) con posible otorrea, hipoacusia, \\
& paresia facial, trombosis del seno sigmoideo y absceso endocraneal \\
& Subclase M: Mastoides \\
& Subclase S: Base de cráneo y seno sigmoideo \\
& Subclase J: Articulación témporo-mandibular \\
& Subclase F: Nervio facial
\end{tabular}

cencia de la cubierta ósea del nervio facial en su tercera porción intratemporal.

Debido a la presencia de compromiso del nervio facial se decidió hospitalización para curaciones periódicas, terapia corticoesteroidal y tratamiento antibiótico intravenoso de amplio espectro de forma empírica, con ceftazidima $1 \mathrm{~g}$ cada 8 horas más metronidazol $500 \mathrm{mg}$ cada 8 horas. Debido a la buena evolución del paciente, se decidió el alta hospitalaria a los 7 días, continuando tratamiento oral hasta completar 14 días. Además, desde el diagnóstico se indicó terapia tópica en el CAE con solución de ciprofloxacino/dexametasona.

El paciente presentó una respuesta clínica favorable, con regresión de la paresia facial. Un mes después se encontraba con movilidad HouseBrackmann 2, sin otros síntomas. Hasta el momento, el paciente ha rechazado la resolución quirúrgica definitiva mediante reconstrucción de la pared del CAE, indicada según se discutirá más adelante.

\section{FACTORES ETIOLÓGICOS}

El 2006, un grupo de investigadores dirigidos por Owen realizó una descripción de los factores etiológicos involucrados en el CC. Los clasificó en primarios - de causa desconocida- con una prevalencia de $52 \%$, y secundarios con $48 \%$. Éstos se subdividen en posoperatorios, postraumáticos, posinflamatorios y posradiación. Por último describe un tercer grupo de colesteatomas asociado a atresia congénita del $\mathrm{CAE}^{3}$.

Las causas de la acumulación patológica de tejido epitelial en el CAE no están bien definidas y se han planteado diferentes hipótesis. Una de ellas es una alteración de la migración epitelial en la membrana timpánica. Normalmente el epitelio de queratina migra desde la membrana timpánica hacia el CAE siguiendo la dirección de los vasos sanguíneos, como mecanismo de autolimpieza del conducto auditivo. Se ha planteado como factor etiológico de la alteración de la migración una hipoperfusión de la membrana timpánica4.

También se ha descrito que existiría una pérdida de la migración normal debida al envejecimiento del epitelio de la pared del CAE, y se ha reportado que el CC es más frecuente en pacientes adultos mayores ${ }^{5}$.

Además, la acumulación de restos de queratina podría aumentar la actividad proliferativa del epitelio queratinizante en la membrana timpánica y piel del $\mathrm{CAE}^{6}$.

\section{PRESENTACIÓN CLÍNICA}

Aunque en ambos casos encontramos detritus epitelial ocupando parcial o totalmente el CAE, existen diferencias semiológicas entre las dos entidades. 


\section{Keratosis obturans}

Se presenta habitualmente en personas adultas jóvenes con hipoacusia de conducción de instalación aguda, otalgia intensa, CAE ensanchado, membrana timpánica engrosada y rara vez otorrea. El dolor y la hipoacusia son secundarias a la acumulación de queratina en el $\mathrm{CAE}^{7}$.

Al examen físico, la piel del CAE se encuentra inflamada, incluyendo la que recubre la membrana timpánica ${ }^{3}$. El compromiso bilateral es más frecuente en los niños ${ }^{8}$.

Un estudio antiguo asoció la $\mathrm{KO}$ a sinusitis 0 bronquiectasias en el $77 \%$ casos de los niños y al $20 \%$ casos de los adultos ${ }^{9}$; lo que quizás podría explicarse por una dificultad en la migración epitelial.

\section{Colesteatoma del CAE}

Se presenta más frecuentemente en pacientes ancianos y no se asocia a otras enfermedades sistémicas. Clínicamente, los pacientes refieren otorrea y dolor unilateral crónico. La audición podría estar levemente comprometida o normal ${ }^{7}$. Al examen se observa una úlcera secundaria a la invasión de tejido escamoso en un área localizada de la pared del CAE. La membrana timpánica se observa normal ${ }^{10}$.

\section{IMAGENOLOGÍA}

En ambas patologías, el examen imagenológico de elección es la tomografía computarizada (TC) de oídos.

\section{Keratosis obturans}

En la KO se observa un CAE amplio debido a la remodelación ósea en un patrón circunferencial, y no se observa osteonecrosis ${ }^{11}$.

\section{Colesteatoma deI CAE}

En la TC de oídos se observa una masa con densidad de tejidos blandos asociada a erosión ósea focal localizada en la cara posteroinferior del CAE, con secuestro óseo. En ocasiones se puede identificar un área adyacente con osteonecrosis y periostitis.
Algunos autores señalan que dichos hallazgos, juntos a la pérdida epitelial focal, son los que mejor permiten diferenciar entre éste y la K0 ${ }^{7,11}$.

\section{ANATOMÍA PATOLÓGICA}

Las diferencias clínicas y radiológicas discutidas anteriormente tienen un correlato histopatológico que permite explicar el comportamiento clínico distintivo de estas dos condiciones.

\section{Keratosis obturans}

Histológicamente, el patrón de organización de las escamas de queratina es circular, donde las capas más antiguas se ubican centralmente y comprimen el CAE remodelándolo circunferencialmente. Además se observa una marcada inflamación y dilatación vascular en el tejido subepitelial del $\mathrm{CAE}^{12}$.

\section{Colesteatoma del CAE}

En el CC hay necrosis ósea localizada y secuestro del hueso subyacente; característica ausente en la $\mathrm{KO}$. Histológicamente, la queratina deriva de un saco y no presenta un patrón de organización definido, distribuyéndose de manera azarosa en todas las direcciones en el $\mathrm{CAE}^{7,12}$.

El 2005, Naim y cols presentaron una clasificación en estadios para el $\mathrm{CC}^{13}$, según hallazgos histopatológicos y manifestaciones clínicas (Tabla 1).

\section{COMPLICACIONES}

Las complicaciones ocurren generalmente en relación a la extensión de la lesión y a la destrucción ósea. También se han descrito infecciones locales graves como la otitis externa necrotizante, producida habitualmente por Pseudomonas aeruginosa; que se presenta con mayor frecuencia en pacientes inmunosuprimidos y diabéticos ${ }^{10}$.

Se han descrito complicaciones como parálisis facial, erosión osicular y fístulas laberínticas. Incluso, la erosión puede comprometer medialmente la base del cráneo hasta el foramen yugular ${ }^{11,14}$.

Ya la mayoría de las complicaciones se deben al compromiso erosivo del hueso subyacente, se 
asocian más frecuentemente en el CC. Aun así, Saunders y cols describieron complicaciones atribuibles a erosión ósea en 3 pacientes con $\mathrm{KO}^{15}$.

\section{TRATAMIENTO}

En el manejo de la KO y del CC se debe considerar la fisiopatología detrás de ambos, ya que permite tomar decisiones racionales en base al comportamiento esperado. Discutiremos las alternativas terapéuticas más aceptadas.

\section{Keratosis obturans}

En general se recomienda el desbridamiento periódico de los restos epiteliales del CAE junto con la aplicación de antibióticos tópicos en caso de infección ${ }^{7}$. La frecuencia de la aspiración del tapón de queratina va a estar determinada por los síntomas, principalmente por la hipoacusia².

\section{Colesteatoma del CAE}

El tratamiento del CC va a estar determinado por la gravedad y naturaleza de los síntomas, la extensión de la enfermedad y la presencia de osteonecrosis. Inicialmente el tratamiento puede ser conservador, indicado para lesiones limitadas con síntomas menores como plenitud aural y otalgia. Esto incluye la realización de limpiezas seriadas con desbridamiento del tejido necrótico, y antibióticos tópicos en caso de infecciones locales ${ }^{5,16}$.

Se recomienda tratamiento quirúrgico en caso de presencia de destrucción ósea, extensión al oído medio o mastoides, pérdida auditiva significativa, secreción crónica que no ceda con el tratamiento médico y presencia de complicaciones ${ }^{17}$. El objetivo de la cirugía es extirpar por completo el tumor y el hueso necrótico; de esta manera se evita la progresión y la recidiva. La técnica quirúrgica dependerá del tamaño de la osteonecrosis, la erosión y experiencia del cirujano ${ }^{7}$.

Algunos autores aconsejan rellenar el defecto óseo para restaurar el contorno del CAE. Lee y cols presentaron una técnica en la cual utilizan un colgajo pediculado de músculo temporal, cubierto con cartílago e injerto de piel retroauricular para regularizar el conducto ${ }^{18}$.

El 2010, Shin y cols presentaron un sistema de estadificación del CC según las hallazgos clínicos y tomográficos para poder definir una conducta terapéutica ${ }^{19}$ (Tabla 2).

\section{CONCLUSIÓN}

La KO y el CC son dos condiciones clínicas infrecuentes que se presentan con retención de epitelio queratinizado descamado en el CAE. Aun así, sus características clínico-patológicas son diferentes por lo que deben ser enfrentadas y tratadas de manera distinta; lo que hace relevante el establecer un adecuado diagnóstico diferencial.

Generalmente, la KO posee una evolución más favorable que el CC, que tiene un comportamiento más agresivo. En el caso de la KO, el tratamiento mediante desbridamiento periódico y manejo de la sobreinfección local suele ser suficiente en la mayoría de los casos. En cambio, el manejo del CC es

Tabla 2. Manejo del colesteatoma del CAE según hallazgos clínicos e imagenológicos (adaptado de Shin y cols ${ }^{19}$ )

\begin{tabular}{|lll|}
\hline Estadio & Definición & Tratamiento \\
\hline I & Compromiso limitado al CAE & Manejo local o canaloplastía \\
II & Invasión a la membrana timpánica y oído medio, además del CAE & Canaloplastía + timpanoplastía \\
III & $\begin{array}{l}\text { Crea un defecto en el CAE y compromete las celdillas aéreas } \\
\text { del hueso mastoideo }\end{array}$ & $\begin{array}{l}\text { Canaloplastía + mastoidectomía } \\
\pm \text { timpanoplastía } \pm \text { reconstrucción de la } \\
\end{array}$ \\
& Compromiso de estructuras fuera del hueso temporal & pared del CAE \\
IV & & Extracción del colesteatoma mediante \\
& & diferentes abordajes \\
\hline
\end{tabular}


más complejo y debe considerarse el tratamiento quirúrgico en casos de gran destrucción ósea.

En el caso clínico presentado, la evolución inicial mediante desbridamiento cuidadoso realizado en la consulta ambulatoria fue favorable. Aun así, de no lograrse una visualización adecuada es aconsejable realizar este procedimiento en pabellón, más aún en presencia de complicaciones. De todas maneras, el manejo definitivo de CC avanzados debiera incluir una reconstrucción del CAE con 0 sin mastoidectomía.

Finalmente, es importante considerar el uso de imágenes, donde la TC de oídos es el examen de elección. Esto permite identificar signos de necrosis, secuestro óseo y periostitis, clasificar el estadio del CC e identificar lesiones erosivas que pueden llevar a complicaciones 0 iatrogenia.

\section{BIBLIOGRAFÍA}

1. Toynbee J. Specimens of molluscum contagiosum developed in the external auditory meatus. Lond Med Gazzette 1850; 46: 811.

2. Piepergerdes MC, Kramer BM, Behnke EE. Keratosis obturans and external auditory canal cholesteatoma. Laryngoscope 1980; 90: 38391.

3. Owen HH, Rosborg J, Gaihede M. Cholesteatoma of the external ear canal: etiological factors, symptoms and clinical findings in a series of 48 cases. BMC Ear Nose Throat Disord 2006; 6: 16.

4. Makino K, Amatsu M. Epithelial migration on the tympanic membrane and external canal. Arch Otorhinolaryngol 1986; 243: 39-42.

5. HoLt JJ. Ear canal cholesteatoma. Laryngoscope 1992; 102(6): 608-13.

6. Park K, Chun ym, Park hJ, Lee yd. Immunohistochemical study of cell proliferation using BrdU labelling on tympanic membrane, external auditory canal and induced cholesteatoma in Mongolian gerbils. Acta Otolaryngol 1999; 119: 874-9.
7. Persaud Ra, Hajioff D, Thevasagayam MS, Wareing MJ, WrIGHT A. Keratosis obturans and external ear canal cholesteatoma: how and why we should distinguish between these conditions. Clin Otolaryngol Allied Sci 2004; 29: 577-81.

8. BLACK JI, CHAYTOR RG. Wax keratosis in children's ears. Br Med J 1958; 2: 673-5.

9. Morrison AW. Keratosis obturans. J Laryngol Otol 1956; 70: 317-21.

10. Negreiros J, Oliveira HF, Neves CA, Oliveira CA. External Auditory Canal Cholesteatoma. Int Adv Otol 2009; 5: 391-3.

11. Heilbrun ME, Salzman KL, Glastonbury CM, Harnsberger HR, Kennedy RJ, Shelton C. External auditory canal cholesteatoma: clinical and imaging spectrum. AJNR Am J Neuroradio/2003; 24: 751-6.

12. Naiberg J, Berger G, Hawke M. The pathologic features of keratosis obturans and cholesteatoma of the external auditory canal. Arch Otolaryngol 1984; 110: 690-3.

13. Naim $R$, Linthicum $F$ Jr, Shen T, Bran G, Hormann K. Classification of the external auditory canal cholesteatoma. Laryngoscope 2005; 115: 455-60.

14. Malcolm PN, Francis IS, Wareing MJ, Cox TC. CT appearances of external ear canal cholesteatoma. Br J Radiol 1997; 70: 959-60.

15. Saunders nC, Malhotra R, Biggs N, Fagan Pa. Complications of keratosis obturans. J Laryngol Otol 2006; 120: 740-4.

16. Vrabec JT, Chaljub G. External canal cholesteatoma. Am J Otol 2000; 21: 608-14.

17. Anthony PF, Anthony WP. Surgical treatment of external auditory canal cholesteatoma. Laryngoscope 1982; 92: 70-5.

18. Lee JH, Jung SH, Park CH, Hong SM. Extensive external auditory canal cholesteatoma in the infratemporal area without mastoid involvement: use of a new surgical technique. Ear Nose Throat J 2010; 89: 438-42.

19. SHIN SH, SHIM JH, LeE HK. Classification of external auditory canal cholesteatoma by computed tomography. Clin Exp Otorhinolaryngol 2010; 3: 24-6. 